\title{
Billing Practices Among US Tobacco Use Treatment Providers
}

\author{
Erika Litvin Bloom, PhD, Michael V. Burke, EdD, Chris Kotsen, PsyD, CTTS, \\ Adam O. Goldstein, MD, MPH, Carol Ripley-Moffitt, MDiv, CTTS, Michael B. Steinberg, MD, MPH, \\ Mary Dailey, CTTS, Laura E. Hunt, BS, and Matthew P. Bars, MS, CTTS
}

Objectives: The US Affordable Care Act (ACA) now requires almost all health insurance plans to cover tobacco use treatment (TUT), but TUT remains underutilized.

Methods: We conducted an anonymous online survey of US TUT providers in 2016 regarding their billing practices.

Results: Participants $(n=131)$ provided services primarily in medical and behavioral health settings and were from a variety of professions. Most provided intensive individual ( $>15$ minutes per session) and/or group counseling. Although most reported that their organization accepted at least 1 form of insurance, only $34 \%$ reported that TUT services were billed, with about equal proportions endorsing billing under their own independent tax ID and "incident to" billing under a supervisor. Half of billers (52\%) reported using at least 1 Current Procedural Terminology code. The most common codes were 99406 and 99407 , but 18 unique codes were specified. Themes of qualitative responses $(n=101)$ included concern about how to initiate and sustain adequate reimbursement, and experiences with billing not being "worth" the time or effort.

Conclusions: Overall, results demonstrate a need for providers, administrators, and billing managers to work collaboratively. Even with the ACA mandate, and consistent with prior reports, reimbursement rates may be inadequate for intensive counseling. Areas for advocacy include recognizing that TUT requires similar intensity,

From the Alpert Medical School of Brown University (ELB); Rhode Island Hospital (ELB, LEH); Mayo Clinic College of Medicine and Nicotine Dependence Center (MVB); Steeplechase Cancer Center at Robert Wood Johnson Barnabas Health University Hospital Somerset (CK); University of North Carolina at Chapel Hill School of Medicine (AOG, CR-M); Rutgers Robert Wood Johnson Medical School (MBS); Florida State University College of Medicine Tobacco Program (MD); Fire Department of the City of New York Tobacco Treatment Program, IQuit Smoking at Jersey City Medical Center RWJ Barnabas (MPB); Business Practices Committee of the Association for the Treatment of Tobacco Use and Dependence (ATTUD) (ELB, MVB, CK, AOG, CR-M, MBS, MD, MPB).

Received for publication October 19, 2017; accepted May 1, 2018.

Funding: This work was supported by Lifespan (Parent Corporation of Rhode Island Hospital).

Conflicts of interest: Dr Bloom has been a consultant for WayBetter, Inc. for work unrelated to this manuscript.

Send correspondence to Erika Litvin Bloom, PhD, Rhode Island Hospital, Division of General Internal Medicine-Research, 593 Eddy St., Providence, RI 02903. E-mail: erika_bloom@brown.edu

ISSN: $1932-0620 / 18 / 1205-0381$

DOI: 10.1097/ADM.0000000000000423 expertise, and reimbursement as other substance use disorders and chronic medical conditions; giving Tobacco Treatment Specialists the ability to bill independently; and improving coordination between intensive therapies validated in research and "real-world" logistics.

Key Words: billing, Current Procedural Terminology (CPT), intensive tobacco cessation counseling, smoking cessation, tobacco treatment

(J Addict Med 2018;12: 381-386)

$T$ obacco use persists as the leading cause of preventable death and disability in the United States (U.S. Department of Health and Human Services, 2014). As of 2010, an estimated $8.7 \%$ of annual healthcare spending was attributable to smoking (Xu et al., 2015). Tobacco use treatment (TUT) is most effective when patients receive at least 4 sessions of counseling that last at least 20 minutes each, and total counseling duration is at least 90 minutes per quit attempt (Fiore et al., 2008; Siu and U.S. Preventive Services Task Force, 2015). However, TUT has long been underutilized (Bernstein et al., 2013; Ku et al., 2016).

In 2014, the Affordable Care Act (ACA) began mandating coverage of TUT as a Preventive Service, including all United States (US) Food and Drug Administration (FDA)approved medications and at least 8 sessions of counseling (individual and group) (4 sessions per quit attempt, 2 attempts per year), by most Medicaid, marketplace, and employersponsored health insurance plans. Medicare has the same individual counseling coverage mandate (group is excluded), but is only required to cover prescription medications. Despite this new ACA mandate, results of the 2015 US National Health Interview Survey (NHIS) revealed that only $31.2 \%$ of smokers said they used treatment when trying to quit $(6.8 \%$ counseling, 29.0\% medication, $4.7 \%$ both) (Babb et al., 2017).

Many provider and systems-level barriers to implementing TUT have been identified (eg, Agaku et al., 2015). An important practical barrier to implementing the ACA mandate that has received less attention is TUT reimbursement policy. Issues include a perceived lack of reimbursement for counseling, inadequate reimbursement at a macro/systems level for treatment, and/or lack of knowledge regarding coding and documentation requirements to receive reimbursement (Bernstein et al., 2013; Agaku et al., 2015; Ku et al., 2016; Leone 
et al., 2016; Nolan and Warner, 2017). Indeed, the ACA does not specify TUT coding requirements and there are numerous ways in which TUT may be coded and documented, which may be contributing to confusion and lack of action (Leone et al., 2016). Leone et al. (2016) have developed a useful TUT billing resource for physicians. Leone et al. also provide a brief description of "incident to" billing in which a nonphysician provider bills under the supervising physician's name. Additionally, a similar "physician extender" model of delivering TUT with clinician-prescriber teams has been proposed and successfully implemented (Burke et al., 2015).

However, many individuals in the US who provide counseling to tobacco users are not physicians nor are they supervised directly by a physician. In 1999, Massachusetts established a Tobacco Treatment Specialist (TTS) training and certification program in response to a need to improve and standardize tobacco treatment services funded by the Massachusetts Tobacco Control Program (MTCP) (Pbert et al., 2000). This TTS model was eventually expanded to other states. In 2008, the Council for Tobacco Treatment Training Programs (the Council; www.ctttp.org) was established to accredit programs that train TTSs. Accredited programs must cover 11 Tobacco Dependence Treatment Core Competencies (Sheffer et al., 2015). Clinicians who attend TTS training programs come from multiple disciplines and work in a variety of health care settings. Smokers treated by clinicians with specialist training are more likely to achieve long-term abstinence than smokers treated by clinicians without specialist training. (McDermott et al., 2013).

There are now 19 Council-accredited TTS programs that trained 1770 clinicians in 2016. Clinicians who complete a Council-accredited TTS training program and 240 hours of practice may apply for the National Certificate of Tobacco Treatment Practice (NCTTP; https://www.naadac.org/ NCTTP). The number of Council-accredited programs and clinicians trained is expected to increase substantially with the establishment of this national credential (NCTTP) (the Council, personal communication, March 2018). Data on the educational background and professions of individuals who attended 11 of the 19 TTS training programs in 2016 that suggest that less than half are likely to be eligible to bill insurance (the Council, personal communication, March 2018). Common professions of TTSs who are unlikely to be qualified to bill independently include nurses, health educators, respiratory therapists, and bachelor-level counselors. If supervised by a physician, some of these individuals may be able to use "incident to" billing.

Given the ACA mandate, an inability to bill insurance could be a significant barrier to provision of TUT by TTS. However, we could not identify any previous studies in which TUT providers were surveyed regarding their experiences with billing after the ACA mandate became law (ie, after 2014). Therefore, the purpose of this study was to anonymously survey US TUT providers to learn about their actual TUT billing practices since 2014. Specifically, we sought to sample a broad cross-section of TUT providers and determine the proportion who engaged in traditional fee-for-service billing versus alternative arrangements, and, among those who did engage in fee-for-service billing, specific practices and codes used.

\section{METHODS}

Participants $(n=131)$ were eligible if they had provided TUT (counseling and/or prescribed medications) in the US within the past year (since summer 2015, study conducted in summer 2016) and the TUT was not exclusively available to participants in a research study (ie, it was in a clinical, workplace, or other community or real-world setting). There was no restriction on age or profession. The study was advertised on e-mail listservs and discussion forums read by US TUT providers, such as the listservs of the Society for Research on Nicotine and Tobacco (SRNT) and the Association for the Treatment of Tobacco Use and Dependence (ATTUD). Advertisements were also sent to lists of individuals who had attended TTS training programs and other professional networks. Advertisements contained a description of the survey, the eligibility criteria for participation, and a link to the survey website. Upon clicking the link, participants viewed an electronic consent form that contained standard consent elements (eg, description of the study, risks and benefits, etc). They had to click on "agree" to indicate that they met the eligibility criteria and consented to participate before they could proceed to answer the survey questions. The consent form indicated that only completed surveys (ie, participant clicked "submit" at end of survey) responses would be analyzed. This study was approved by the Institutional Review Board at Lifespan, the parent corporation of Rhode Island Hospital. The survey took less than 15 minutes to complete. Results were analyzed from 2016 to 2018.

The survey was created and managed using Research Electronic Data Capture (REDCap) (Harris et al., 2009) tools hosted at Lifespan. The REDCap public survey option was used. REDCap public surveys are completely anonymous unless the survey questions ask for identifying information. Participants were asked to specify their demographics, details of their educational and professional background, their work setting(s), years of experience providing TUT, typical hours and number of TUT patients per week, TUT treatment types (eg, counseling formats and styles), whether and which insurances they and/or their organization accepted, whether and how patients were billed and how much patients paid for TUT, whether and which billing codes (ie, Current Procedural Terminology $[\mathrm{CPT}]$ codes) were used for TUT, and whether their organization had a billing manager or team and if so their perception of the billing manager/team's level of knowledge and experience regarding billing and coding for TUT (rated on 5-point scale from "none" to "excellent"). Finally, participants responded to an open-ended question in which they were given the opportunity to: share details about their experiences with billing for TUT (eg, positive experiences, negative experiences, challenges, changes in how they have billed over time) that they thought would be informative for other TUT providers; share information about their billing practices that they believed was important but not captured by the other questions; and if they did not bill, to explain their arrangement. These responses were reviewed and grouped into categories (note: some responses did not fit into any 


\begin{tabular}{lc}
\hline TABLE 1. & \\
\hline Profession & n (\%) \\
\hline Nurse & $20(16 \%)$ \\
Counselor/therapist (Bachelor's or Master's level) & $17(13 \%)$ \\
Respiratory therapist & $14(11 \%)$ \\
Nurse practitioner & $11(9 \%)$ \\
Health educator & $10(8 \%)$ \\
Social worker & $8(6 \%)$ \\
Psychologist & $7(5 \%)$ \\
Physician & $6(5 \%)$ \\
Pharmacist & $5(4 \%)$ \\
Health or wellness coach & $5(4 \%)$ \\
Other* & $26(20 \%)$
\end{tabular}

Data collected online from participants in the USA in 2016.

${ }^{*} \mathrm{n}=10$ within this group identified themselves only as a "Tobacco Treatment Specialist" with no other profession listed.

category and others were assigned to more than one category). At the end of the survey, participants had the option to provide their email address to be entered into a lottery to win one of four Amazon.com \$25 gift cards.

\section{RESULTS}

\section{Participant Characteristics and Work Settings}

The survey was accessed 204 times. Blank and incomplete surveys $(n=69)$ and completed surveys from individuals who were determined to be ineligible $(n=4)$ were excluded, resulting in a final sample of 131 participants. Participants represented diverse professions (Table 1). Overall, 72\% identified as TTS. Participant demographics and TUT details (eg, hours, settings) are presented in Table 2. Most were working in traditional medical and behavioral health settings (Table 2).

\section{Tobacco Use Treatment Formats}

Most participants did not have prescription privileges and therefore only provided counseling; $17 \%$ wrote prescriptions for medication. Among those who did not write prescriptions, $52 \%$ reported working collaboratively with a prescribing provider. Counseling formats included intensive ( $>15$ minutes per session) $(71 \%)$, brief $(5-15$ minutes per session) (67\%), brief advice ( $<5$ minutes per session) $(50 \%)$, and group counseling (48\%). Counseling styles and orientations included motivational interviewing (89\%), cognitivebehavioral therapy (CBT) (47\%), and acceptance and commitment therapy (ACT) (20\%). Another commonly provided service was breath carbon monoxide testing (49\%).

\section{Billing and Coding Practices}

\section{Types of Insurance Accepted and Whether Billing Occurred}

Only $21 \%$ of participants said that their organization did not accept insurance and 6\% did not know (2 participants who selected "I don't know" and at least 1 insurance type were coded as "I don't know."). We excluded these participants $(\mathrm{n}=35)$ from analyses of billing and coding practices. Among the remaining participants $(n=96), 93 \%$ said their organization accepted private insurance, $87 \%$ Medicare, and $91 \%$
Medicaid. However, $46 \%$ of these participants reported that they did not bill for TUT and 8\% did not know whether their TUT was billed or did not answer this question ("nonbillers"; $\mathrm{n}=52$ ). Nonbillers included 13 nurses or nurse practitioners, 2 physicians, 11 social workers or other master's level counselors, 5 health educators, 5 respiratory therapists, 2 pharmacists, and various other professions.

\section{Specific Billing Practices and Patient Costs}

Among the participants who endorsed at least one specific billing practice ("billers"; $n=44,34 \%$ of the total sample of 131$), 43 \%$ reported that they billed independently under their own tax ID and $45 \%$ endorsed "incident to" billing under a supervisor (3 participants endorsed both). Other arrangements included capitated (per person fee) $(11 \%)$ or another contract $(5 \%)$. About half of billers $(52 \%)$ reported that a typical patient did not pay any out-of-pocket cost for TUT (\$0 co-pay), 36\% selected "I don't know" how much patients paid, and most of the rest said typical patients paid a co-pay or had to pay up to their deductible (note: a few participants selected "I don't know" in addition to another option). Finally, 52\% of billers reported using at least $1 \mathrm{CPT}$ code. The most commonly used codes were the smoking and tobacco use cessation counseling codes 99406 (3-10 minutes) and 99407 ( $\geq 10$ minutes), but 18 unique codes were specified (Table 3 ). Billers included 3 physicians, 12 nurses or nurse practitioners, 8 respiratory therapists, 6 psychologists, 3 social workers or other master's level counselors, 3 bachelor's level counselors, and 1 pharmacist. Other billers identified only as TTS or another profession.

\section{Billing Management Knowledge and Experience}

Among participants whose organizations accepted at least 1 form of insurance $(n=96), 82 \%$ reported that they had a billing manager or team. Regarding their billing manager/

\section{TABLE 2. Participant Characteristics}

\begin{tabular}{lccc}
\hline & $\begin{array}{c}\text { Mean } \\
\text { or \% }\end{array}$ & SD & Range \\
\hline Age, yrs & 46.6 & 12.5 & $23-68$ \\
Sex (female) & $87 \%$ & & \\
Education level & $7 \%$ & & \\
$\quad$ Less than bachelor's degree & $11 \%$ & & \\
$\quad$ Bachelor's degree & $27 \%$ & & \\
$\quad$ Master's degree & $46 \%$ & & \\
$\quad$ Doctorate degree & $17 \%$ & & \\
Years of experience in TUT & 7.6 & 6.7 & $<1-36$ \\
Hours per week providing TUT & 13.4 & 12.3 & $<1-40$ \\
Patients per week seen for TUT & 11.5 & 11.5 & $<1-54$ \\
Proportion of working hours spent on TUT & $34 \%$ & $33 \%$ & $<1 \%-100 \%$ \\
Work settings & & &
\end{tabular}

Work settings

$\begin{array}{lc}\text { Outpatient primary care } & 44,34 \% \\ \text { Inpatient medical } & 42,32 \% \\ \text { Outpatient specialty medical } & 38,29 \% \\ \text { Worksite wellness } & 34,26 \% \\ \text { Outpatient psychiatric/behavioral health } & 18,14 \% \\ \text { Lung cancer screening program } & 17,13 \% \\ \text { Outpatient substance use } & 14,11 \% \\ \text { Inpatient psychiatric } & 9,7 \% \\ \text { Inpatient/residential substance use } & 3,2 \% \\ \text { Other (eg, phone, community-based } & 33,25 \%\end{array}$

program)

Data collected online from participants in the USA in 2016. 
TABLE 3. Current Procedural Terminology (CPT) Codes Used and Descriptors

\begin{tabular}{lcl}
\hline Code & Participants (\#) & \multicolumn{1}{c}{ Descriptor } \\
\hline G0436* & 5 & Smoking and tobacco use cessation counseling 3-10 min, asymptomatic \\
G0437* & 6 & Smoking and tobacco use cessation counseling $>10$ min, asymptomatic \\
90791 & 1 & Psychiatric diagnostic interview without medical services \\
90834 & 1 & Individual psychotherapy (45 min) \\
90837 & 1 & Individual psychotherapy (60 min) \\
90853 & 1 & Group psychotherapy \\
94250 & 3 & Expired gas collection (breath carbon monoxide testing) \\
96150 & 3 & Health and behavior assessment, each 15 min \\
96151 & 1 & Health and behavior re-assessment, each 15 min \\
96152 & 3 & Health and behavior intervention, each 15 min, individual \\
96153 & 1 & Health and behavior intervention, each 15 min, group \\
99401 & 2 & Preventive medicine counseling and/or risk factor intervention(s) provided to an individual, 15 min \\
99402 & 3 & Preventive medicine counseling and/or risk factor intervention(s) provided to an individual, 30 min \\
99403 & 3 & Preventive medicine counseling and/or risk factor intervention(s) provided to an individual, 45 min \\
99404 & 3 & Preventive medicine counseling and/or risk factor intervention(s) provided to an individual, 60 min \\
99406 & 13 & Smoking and tobacco use cessation counseling 3-10 min \\
99407 & 18 & Smoking and tobacco use cessation counseling >10 min \\
D1320 & 1 & Tobacco counseling for the control and prevention of oral disease (dental billing code) \\
\hline
\end{tabular}

Data collected online from participants in the USA in 2016. One $(\mathrm{n}=1)$ participant who said "I don't know" whether patients are billed and 1 who stated that their organization didn't accept insurance and endorsed "other" for billing practice each endorsed a single CPT code (99407 and D1320, respectively; these codes are included in the table).

${ }^{*}$ These codes were discontinued for services provided after 10/1/16; 99406 and 99407 may now be used for all patients.

team's knowledge of and experience with TUT billing and coding, 35\% said "none," 29\% "a little," 16\% "some," 13\% "good," and 6\% said "excellent." All billers $(\mathrm{n}=44)$ endorsed having a billing manager/team, and with regard to the manager/team's knowledge and experience, $18 \%$ said "none," 36\% "a little," 27\% "some," 14\% "good," and 5\% said "excellent."

\section{Qualitative Responses}

Of the 131 participants, 101 provided a response to the open-ended question about their billing experience. Fortythree $(n=43)$ of the 101 indicated that they did not engage in traditional fee-for-service billing; many stated that TUT was provided to patients at no cost and their salary was covered by their employer (eg, cancer center, community-based organization, workplace wellness, and/or human resources benefit) and/or TUT services were "bundled" with other services (eg, inpatient setting, rehab programs). Three of these 43 participants noted that they could not bill as they were not a recognized "provider." Twenty-four $(\mathrm{n}=24)$ participants stated that their salary and/or TUT program was covered by a grant or other federal funding; most of them indicated that TUT was provided to patients at no cost. Three participants said that they charged patients directly (ie, self-pay program). Nine participants, including 6 of the grant-funded participants, said that they were in the process of initiating billing or were exploring and/or interested in finding sustainable funding for their TUT programs. Finally, 16 participants expressed difficulties with and/or frustration regarding billing, generally pertaining to being unable to determine how to bill correctly and/or sustainably despite seeking that information; or beliefs that billing was not "worth it" (eg, time involved to set up and manage billing would be less than reimbursement received) based on their experiences. Some examples include the following:

"I find it VERY frustrating that there is no place that a person doing the billing can go to access the necessary codes, what each code will pay, etc. for Medicaid reimbursement."

\begin{abstract}
"Ifeel overwhelmed trying to create billing for my services even in partnership with a provider. It is my hope that TTSs will be seen as stand alone providers in the future with the ability to charge directly for our time."
\end{abstract}

"We had been billing in the past but revenue was insufficient to make ongoing billing possible. Therefore, the organization no longer bills for tobacco treatment."

"It seems like my organization has never tried to bill for group counseling for smoking cessation before, and they are often confused as to how to bill most effectively. They also will occasionally accept patients for intake whose insurances will pay for group counseling for mental health but not for tobacco use, so that's frustrating when patients are billed at self-pay rates when initially told they were covered. It's very difficult to launch a self-sustaining group. If we did not take insurance, and the system was more inclined to provide these preventive services at little to no cost, everybody would be much more satisfied, more people would get treatment, cost savings would be very high in my view, and the group would be filled. Right now, even with some institutional funding I have received to launch it, it is very hard to get more than 3-4 patients coming regularly to group - this makes the group statistically not worth my time because I could bill that hour for a different service and get more credits. So, that is frustrating."

\section{DISCUSSION}

In this study, 131 US providers of TUT were surveyed anonymously regarding whether they billed insurance for TUT, and if so, their billing practices. Our goal of sampling 
a broad cross-section of TUT providers appeared to be met. Most worked in medical and/or behavioral health settings where fee-for-service billing presumably could occur.

\section{Billing Prevalence and Specific Billing Practices}

Among participants whose organizations accepted at least 1 form of insurance and therefore had the potential ability to bill (96 of the 131 participants), almost half reported that the TUT services they provided were not billed ("nonbillers"). However, lack of billing could not be attributed to profession (ie, their profession did not qualify them to bill). Both the nonbillers and billers groups included a substantial number of participants from professions that would likely be qualified to bill.

Among the billers, roughly equal proportions said that they billed independently under their own independent tax ID or "incident to" under a supervisor. Although the ACA mandate for TUT was intended to ensure that patients would not pay any outof-pocket cost for TUT counseling, only half of billers reported that a typical patient did not pay. Others indicated that patients typically paid co-pays or did not know how much patients paid.

\section{CPT Coding}

Among billers, half reported using at least 1 specific CPT code. The most commonly used codes were 99406 and/or 99407. Reimbursement rates for these codes are relatively low, reflecting their intended application for brief counseling $(<15$ minutes) sessions. Yet, most participants in the sample as a whole and in the subsample endorsing these codes reported providing intensive counseling $(>15$ minutes per session). Therefore, even with the ACA mandate, it remains unclear if intensive individual counseling (ie, 20-60 minutes per session) and group counseling (typically 60-90 minutes per session) that may be required to achieve abstinence among severely dependent tobacco users is adequately reimbursed.

\section{Qualitative Findings}

In the sample as a whole, participants indicated in their open-ended responses that their time spent providing TUT services was funded by a variety of alternative arrangements to fee-for-service billing. Some were supported by time-limited grants; in some cases, it was uncertain whether they would be able to continue providing TUT services after the grant ended. Most importantly, a substantial number were interested in billing insurance, but had difficulty obtaining accurate information about how to bill and/or had frustrating experiences that led them to determine that billing was not "worth it."

\section{Implications for Practice and Policy}

Although our participants reported providing intensive counseling, our survey results suggest that codes for brief interventions are most commonly used. Clinical practice guidelines for TUT (Fiore et al., 2008) indicated over a decade ago that "it is difficult to accurately code and receive reimbursement for these services... and different payors, may require different codes for reimbursement" (page 231). Questions remain whether insurers are not fully covering the most effective, updated, evidence-based counseling treatments, and/or if some clinicians and their billing teams are not aware of higher intensity treatment codes.
Our results confirm a need for providers, administrators, and billing managers to work collaboratively with insurance companies to advocate for adequate reimbursement for intensive TUT counseling. Williams et al. (2016) highlight the "stark contrast" between levels of care and treatment options for non-nicotine substance use disorders (SUDs), which have been organized into a formal algorithm by the American Society of Addiction Medicine (ASAM) versus tobacco use disorder, for which low-intensity treatment is typically offered regardless of severity. Furthermore, Williams et al. speculate that some insurance companies may not reimburse providers for psychotherapy visits (eg, CPT codes 90832 and 90834) with a primary diagnosis of tobacco use disorder, despite that tobacco use disorders are included in the Diagnostic and Statistical Manual of Mental Disorders, Fifth edition (American Psychiatric Association, 2013) and the International Statistical Classification of Diseases and Related Health Problems, 10th Revision (World Health Organization, 1992); other SUDs are reimbursable with these codes; the primary diagnosis of tobacco use disorder and TUT services are now reclassifed also as preventive care services; and Public Health Service guidelines (Fiore et al., 2008) indicated traditional psychiatric billing codes exist for TUT.

\section{Limitations}

This study had several significant limitations. First, we recruited a convenience, nonrandom sample and to maximize privacy did not ask participants to specify their location within the US. Therefore, participants may not be representative of US TUT providers. Nevertheless, some participants submitted their professional e-mail address for the gift card lottery from which we could determine their likely location. Participants appeared to be broadly distributed throughout the US. Second, given the nature of the study, TUT providers who have encountered billing challenges may have been more eager and likely to participate. At the same time, 1 of our primary findings was that the majority of participants did not bill for their TUT services. Finally, while we were pleasantly surprised at the diversity of the sample with regard to professions and settings, the disadvantage of such diversity is that sample sizes within professions and settings were too small to evaluate whether there were any differences in billing practices and experiences between professions and settings.

\section{CONCLUSIONS}

In conclusion, we found that most TUT providers were not using fee-for-service billing, at least partly out of complexities involved in billing. In some cases, services may be unsustainable as temporary grant funding sources are exhausted. Many participants were interested in fee-for-service billing but had encountered frustrating barriers and challenges in initiating and/or sustaining billing procedures. Areas for advocacy include recognizing that TUT requires similar intensity, expertise, and reimbursement as other SUDs and chronic medical conditions (Bernstein et al., 2013; Williams et al., 2016); giving TTS the ability to bill independently; and improving coordination between the intensive therapies developed and validated in research settings (ie, 
at least 4 individual counseling sessions per quit attempt lasting at least 20 minutes each) (Siu and U.S. Preventive Services Task Force, 2015) and "real-world" CPT codes and logistics. A parallel study of major private insurers' payment experiences of claims for TUT by clinician type and intensity of treatment (ie, brief, intensive, individual, and group) would be especially illustrative and provide additional guidance for developing best billing practices and lowering overall healthcare costs attributable to tobacco use.

\section{ACKNOWLEDGMENTS}

The authors would like to acknowledge Christine Sheffer, PhD, and Denise Jolicoeur, MPH, CHES, for providing the authors with suggested text and references to include in our manuscript to describe the history and current status of Tobacco Treatment Specialists (TTS) and data from the Council on Tobacco Treatment Training Programs (the Council).

\section{REFERENCES}

Agaku IT, Olaiya O, Quinn C, et al. A mixed-methods assessment of a brief smoking cessation intervention implemented in Ohio public health clinics, 2013. Matern Child Health J 2015;19:2654-2662.

American Psychiatric Association. Diagnostic and Statistical Manual of Mental Disorders, 5th edition (DSM-V). Washington, DC: American Psychiatric Association; 2013.

Babb S, Malarcher A, Schauer G, et al. Quitting smoking among adults: United States, 2000-2015. MMWR Morb Mortal Wkly Rep 2017;65:1457-1464.

Bernstein SL, Yu S, Post LA, et al. Undertreatment of tobacco use relative to other chronic conditions. Am J Public Health 2013;103:e59-e65.

Burke MV, Ebbert JO, Schroeder DR, et al. Treatment outcomes from a specialist model for treating tobacco use disorder in a medical center. Medicine (Baltimore) 2015;94:e1903.

Fiore MC, Jaen CR, Baker TB, et al. Treating Tobacco Use and Dependence: 2008 Update. Clinical Practice Guideline. Rockville, MD: U.S. Department of Health and Human Services. Public Health Service; 2008.
Harris PA, Taylor R, Thielke R, et al. Research electronic data capture (REDCap): a metadata-driven methodology and workflow process for providing translational research informatics support. J Biomed Inform 2009;42:377-381.

$\mathrm{Ku}$ L, Bruen BK, Steinmetz E, et al. Medicaid tobacco cessation: big gaps remain in efforts to get smokers to quit. Health Aff (Millwood) 2016; 35:62-70.

Leone FT, Evers-Casey S, Mulholland MA, et al. Integrating tobacco use treatment into practice: billing and documentation. Chest 2016;149: 568-575.

McDermott MS, Beard E, Brose LS, et al. Factors associated with differences in quit rates between "specialist" and "community" stop-smoking practitioners in the english stop-smoking services. Nicotine Tob Res 2013;15: 1239-1247.

Nolan MB, Warner DO. Perioperative tobacco use treatments: putting them into practice. BMJ 2017;358:j3340.

Pbert L, Ockene JK, Ewy BM, et al. Development of a state wide tobacco treatment specialist training and certification programme for Massachusetts. Tob Control 2000;9:372-381.

Sheffer CE, Payne T, Ostroff JS, et al. Increasing the quality and availability of evidence-based treatment for tobacco dependence through unified certification of Tobacco Treatment Specialists. J Smoking Cessation 2015;11: 229-235.

Siu AL, U.S. Preventive Services Task Force. Behavioral and pharmacotherapy interventions for tobacco smoking cessation in adults, including pregnant women: U.S. Preventive Services Task Force Recommendation Statement. Ann Intern Med 2015;163:622-634.

U.S. Department of Health and Human Services. The Health Consequences Of Smoking: 50 Years of Progress: a Report of the Surgeon General. Atlanta, GA: U.S. Department of Health and Human Services, Centers for Disease Control and Prevention, National Center for Chronic Disease Prevention and Health Promotion, Office on Smoking and Health; 2014.

Williams JM, Steinberg ML, Kenefake AN, et al. An argument for change in tobacco treatment options guided by the ASAM criteria for patient placement. J Addict Med 2016;10:291-299.

World Health Organization. ICD-10: international statistical classification of diseases and related health problems. Geneva, Switzerland: World Health Organization; 1992.

Xu X, Bishop EE, Kennedy SM, et al. Annual healthcare spending attributable to cigarette smoking: an update. Am J Prev Med 2015;48:326-333. 